Image quality assessment in digital mammography: part I. Technical characterization of the systems

This article has been downloaded from IOPscience. Please scroll down to see the full text article.

2011 Phys. Med. Biol. 564201

(http://iopscience.iop.org/0031-9155/56/14/002)

View the table of contents for this issue, or go to the journal homepage for more

Download details:

IP Address: 155.105.7.44

The article was downloaded on 14/09/2011 at 09:07

Please note that terms and conditions apply. 


\title{
Image quality assessment in digital mammography: part I. Technical characterization of the systems
}

\author{
N W Marshall ${ }^{1}$, P Monnin ${ }^{2,3}$, H Bosmans ${ }^{1}$, F O Bochud ${ }^{2}$ and \\ F R Verdun ${ }^{2}$ \\ ${ }^{1}$ UZ Gasthuisberg, Department of Radiology, Herestraat 49, 3000 Leuven, Belgium \\ 2 University Institute for Radiation Physics (IRA), CHUV, UNIL, Grand Pré 1, 1007 Lausanne, \\ Switzerland \\ ${ }^{3}$ Haute Ecole Cantonale Vaudoise de la Santé (HECVSanté), Filière TRM, \\ Avenue de Beaumont 21, 1011 Lausanne, Switzerland \\ E-mail: nicholas.marshall@uz.kuleuven.ac.be
}

Received 1 March 2011, in final form 4 May 2011

Published 23 June 2011

Online at stacks.iop.org/PMB/56/4201

\begin{abstract}
In many European countries, image quality for digital x-ray systems used in screening mammography is currently specified using a threshold-detail detectability method. This is a two-part study that proposes an alternative method based on calculated detectability for a model observer: the first part of the work presents a characterization of the systems. Eleven digital mammography systems were included in the study; four computed radiography (CR) systems, and a group of seven digital radiography (DR) detectors, composed of three amorphous selenium-based detectors, three caesium iodide scintillator systems and a silicon wafer-based photon counting system. The technical parameters assessed included the system response curve, detector uniformity error, pre-sampling modulation transfer function (MTF), normalized noise power spectrum (NNPS) and detective quantum efficiency (DQE). Approximate quantum noise limited exposure range was examined using a separation of noise sources based upon standard deviation. Noise separation showed that electronic noise was the dominant noise at low detector air kerma for three systems; the remaining systems showed quantum noise limited behaviour between 12.5 and $380 \mu \mathrm{Gy}$. Greater variation in detector MTF was found for the DR group compared to the CR systems; MTF at $5 \mathrm{~mm}^{-1}$ varied from 0.08 to 0.23 for the CR detectors against a range of $0.16-0.64$ for the DR units. The needle CR detector had a higher MTF, lower NNPS and higher DQE at $5 \mathrm{~mm}^{-1}$ than the powder CR phosphors. DQE at $5 \mathrm{~mm}^{-1}$ ranged from 0.02 to 0.20 for the CR systems, while DQE at $5 \mathrm{~mm}^{-1}$ for the DR group ranged from 0.04 to 0.41 , indicating higher DQE for the DR detectors and needle CR system than for the powder CR phosphor systems. The technical evaluation
\end{abstract}


section of the study showed that the digital mammography systems were well set up and exhibiting typical performance for the detector technology employed in the respective systems.

\section{Introduction}

Before a digital mammography x-ray system can be used for breast screening in many European countries, the system must meet the minimum image quality performance defined in the current edition of the European Guidelines for Quality Assurance in Mammography Screening (European Commission 2006), within the dose limits given in this document. While not an official document that is cast directly in European legislation, these guidelines are extremely influential and have been adopted as de facto minimum performance standards in many European countries (see for example NHSBSP (2009a)).

The image quality standard is specified in terms of threshold-detail detectability for a range of circular discs. The detectability test as presented in the guidelines is used as a system test, rather than a test that focuses solely on x-ray detector performance, as is the case for fluoroscopy detectors in the UK (Hay et al 1985). A range of system factors will therefore influence the ability of a unit to meet the standard in the guidelines; these include $\mathrm{x}$-ray tube potential, tube anode and filter combination, scatter rejection method and imaging performance of the $\mathrm{x}$-ray detector, often specified via the detective quantum efficiency (DQE). Although presented as a system test, threshold contrast-detail detectability test objects often have a limited dynamic range and generate images that are somewhat removed from what can be considered typical patient content in terms of greyscale, spatial frequency and contrast range. Further limitations of this method are examined in the second part of this study.

Along with the choice of operating point for the automatic exposure control (AEC), one of the main parameters determining system imaging performance is the $\mathrm{x}$-ray detector (Aufrichtig 1999, Samei and Flynn 2003). Detector performance can be assessed using quantitative measurements such as the modulation transfer function (MTF), normalized noise power spectrum (NNPS) and DQE (Metz et al 1995, Samei et al 2006, Dobbins et al 2006). While not the final determinant of system image quality, these parameters have considerable impact on the quality of images produced by an imaging system (Aufrichtig 1999, Marshall 2006a). Part two of this work proposes a model observer approach for use in screening mammography image quality specification and correlates the calculated detectability index with the standard metric, that of threshold-detail detectability measured using the CDMAM test object. Given that these are system specific rather than detector specific metrics of image quality, it is important to establish the performance of the detectors using standard quantitative metrics. This will help to isolate elements of system performance related to the intrinsic detector quality from those related to the AEC operating point, x-ray contrast and scatter rejection methods. Furthermore, the first part of the study provides a useful comparison of a range of currently available mammography $x$-ray detectors. The aims of the paper were therefore to characterize technical performance using quantitative measures such as response curve, detector uniformity error, MTF, NNPS and DQE for the $11 \mathrm{x}$-ray detectors in the study. Finally, data presented in this part should confirm whether the performance for a given detector could be considered typical when evaluated by the threshold-detail detectability method and the calculated detectability index, as described in the second part of this work. 
Table 1. Characteristics of the $x$-ray detectors assessed in this study along with the detector response type and fit coefficients.

\begin{tabular}{|c|c|c|c|c|c|c|}
\hline $\begin{array}{l}\text { Detector } \\
\text { name }\end{array}$ & Technology & $\begin{array}{l}\text { Pixel } \\
\text { pitch } \\
(\mu \mathrm{m})\end{array}$ & $\begin{array}{l}\text { Pixel } \\
\text { matrix }\end{array}$ & $\begin{array}{l}\text { Detector } \\
\text { response } \\
\text { curve }\end{array}$ & $A$ & $B$ \\
\hline Agfa MM 3.0R & Single-side powder CR & 50 & $4708 \times 5844$ & Power & 695.73 & 0.521 \\
\hline Agfa HM 5.0 & Single-side needle CR & 50 & $4708 \times 5844$ & Power & 702.53 & 0.518 \\
\hline Fuji Profect & Dual-side powder CR & 50 & $3540 \times 4740$ & Logarithmic & -570.46 & 228.18 \\
\hline $\begin{array}{l}\text { Carestream } \\
\text { EHR-M3 }\end{array}$ & Single-side powder CR & 48.5 & $3584 \times 4784$ & Logarithmic & 2858.5 & -359.37 \\
\hline Fuji Amulet & a-Se/optical switch & 50 & $3540 \times 4740$ & Logarithmic & -1010.3 & 1874.4 \\
\hline $\begin{array}{l}\text { GE Senographe } \\
\text { 2000D }\end{array}$ & CsI/a-Si TFT switch & 100 & $1914 \times 2294$ & Linear & -3.91 & 10.08 \\
\hline GE Senographe DS & CsI/a-Si TFT switch & 100 & $1914 \times 2294$ & Linear & -12.57 & 8.83 \\
\hline GE Essential & CsI/a-Si TFT switch & 100 & $2394 \times 3062$ & Linear & -12.65 & 7.75 \\
\hline Hologic Selenia & $a-\mathrm{Se} / \mathrm{TFT}$ switch & 70 & $2140 \times 2140$ & Linear & 41.21 & 4.08 \\
\hline Sectra MDM & $\begin{array}{l}\text { Photon counter/ } \\
\text { Si-wafer }\end{array}$ & 50 & $4915 \times 5355$ & Linear & - & - \\
\hline Siemens Inspiration & $a-\mathrm{Se} / \mathrm{TFT}$ switch & 85 & $2658 \times 3318$ & Linear & 50.31 & 3.24 \\
\hline
\end{tabular}

\section{Materials and methods}

\subsection{Digital mammography detectors studied}

Eleven digital mammography systems were included in this study, with examples of all the commercially available detector technologies. Four computed radiography (CR) systems were assessed, including two single-sided readout powder phosphor systems, a dual-sided readout units used with a powder phosphor and a single-sided readout system used with a needle CR phosphor. Of the remaining systems, six used flat-panel detectors. Three of these were indirect conversion units that used a caesium iodide phosphor bonded to a light-sensitive thin film transistor (TFT) array formed from amorphous silicon $(a-\mathrm{Si})$. Two further flat-panel systems were evaluated; these were direct conversion detectors using amorphous selenium $(a-\mathrm{Se})$ in conjunction with a TFT readout array. The final unit utilized a silicon wafer photon counter with pre- and post-breast slit collimation. Basic technical parameters for the systems are given in table 1 .

CR detectors are generally not integrated into a given x-ray mammography system but can be used with x-ray units from different suppliers. Both Agfa CR detectors in this study were used with a Siemens Mammomat 3000 system while the Fuji Profect cassettes were used with a Siemens Mammomat 3000 Nova X-ray system. Images with the Carestream EHR-M3 were acquired with a Trex Benett Contour 2000 unit. CR system performance, in terms of image quality produced for a given mean glandular dose (MGD), therefore depends on the anode/filter (A/F) settings available on X-ray unit with which the cassettes are used. Agfa give explicit A/F recommendations for different X-ray systems while Fuji make no recommendation for the Profect CR system and hence A/F used will often be chosen in conjunction with the local Medical Physics department. The Trex system, used for the Carestream EHR-M3 $\mathrm{CR}$ acquisitions, only had molybdenum/molybdenum (Mo/Mo) A/F available. There is no common A/F setting available between the 11 units and hence a common energy could not be selected for the objective image quality evaluations. 
Table 2. Acquisition factors used for the evaluation along with number of photons $\mathrm{mm}^{-2} \mu \mathrm{Gy}^{-1}$; approximate air kerma at the detector for the AEC mode; air kerma at the detector for the DQE evaluation; detector uniformity error (coefficient of variation $(\%)$ ).

\begin{tabular}{|c|c|c|c|c|c|}
\hline $\begin{array}{l}\text { Detector } \\
\text { name }\end{array}$ & $\begin{array}{l}\text { Tube potential/ } \\
\text { anode/ } \\
\text { filter }\end{array}$ & $\begin{array}{l}q_{0} \\
\left(\mathrm{~mm}^{-2} \mu \mathrm{Gy}^{-1}\right)\end{array}$ & $\begin{array}{l}\text { Air kerma } \\
\text { at detector } \\
\text { for } \mathrm{AEC} \\
\text { mode }(\mu \mathrm{Gy})\end{array}$ & $\begin{array}{l}K \text { for DQE } \\
\text { and NNPS } \\
\text { evaluation } \\
(\mu \mathrm{Gy})\end{array}$ & $\begin{array}{l}\text { Detector } \\
\text { uniformity } \\
\text { error } \\
(\%)\end{array}$ \\
\hline Agfa MM 3.0R & $29 \mathrm{kV} \mathrm{Mo} / \mathrm{Rh}$ & 5662 & 102 & 115 & 13 \\
\hline Agfa HM 5.0 & $29 \mathrm{kV} \mathrm{Mo} / \mathrm{Rh}$ & 5662 & 104 & 115 & 14 \\
\hline Fuji Profect & $27 \mathrm{kV} \mathrm{Mo/Rh}$ & 5462 & 64 & 124 & 9.1 \\
\hline Carestream EHR-M3 & $28 \mathrm{kV} \mathrm{Mo/Mo}$ & 5057 & 65 & 92 & 17 \\
\hline Fuji Amulet & $29 \mathrm{kV} \mathrm{W} / \mathrm{Rh}$ & 6168 & 88 & 96 & 1.4 \\
\hline GE Senographe 2000D & $28 \mathrm{kV} \mathrm{Rh} / \mathrm{Rh}$ & 6118 & 66 & 91 & 2.7 \\
\hline GE Senographe DS & $28 \mathrm{kV} \mathrm{Mo} / \mathrm{Mo}$ & 5057 & 98 & 86 & 2.4 \\
\hline GE Essential & $29 \mathrm{kV} \mathrm{Rh} / \mathrm{Rh}$ & 6248 & 83 & 92 & 2.1 \\
\hline Hologic Selenia & $29 \mathrm{kV} \mathrm{W} / \mathrm{Rh}$ & 6168 & 100 & 104 & 2.7 \\
\hline Sectra MDM & $28 \mathrm{kV} \mathrm{W} / \mathrm{Al}$ & 6249 & 64 & 104 & - \\
\hline Siemens Inspiration & $28 \mathrm{kV} \mathrm{W} / \mathrm{Rh}$ & 6070 & 98 & 102 & 1.2 \\
\hline
\end{tabular}

\subsection{Test equipment}

First, a note on the equipment used for the evaluations. As image data were acquired from $\mathrm{X}$-ray machines located at two locations (Switzerland and Belgium), two sets of test equipment had to be used to acquire these data (two different $2 \mathrm{~mm} \mathrm{Al} \mathrm{filters,} \mathrm{dosemeters} \mathrm{and} \mathrm{MTF}$ edges). The dosemeters were a Radcal monitor (Radcal, Monrovia, USA) used with a $6 \mathrm{~cm}^{3}$ ionization chamber $(10 \times 5-6 \mathrm{M})$ and an RTI Barracuda (Mölndal, Sweden) used with a solid-state multipurpose detector (MPD). Calibration of both devices was traceable to national standards. As an additional check on consistency of the output measurements, the two dosemeters were brought together for comparative measurements on a single mammography system. Output with $2 \mathrm{~mm}$ added $\mathrm{Al}$ in the X-ray beam was measured as a function of tube current-time product (mAs) with the two dosemeters. Two MTF edges were used; a Tungsten square of side $50 \mathrm{~mm}$ and thickness $0.5 \mathrm{~mm}$ and a steel rectangle of dimensions $60 \mathrm{~mm} \times$ $120 \mathrm{~mm}$ and thickness of $1 \mathrm{~mm}$.

\subsection{Detector response}

The first step in the technical characterization was the measurement of the detector response at the tube potential and A/F given in table 2, with a $2 \mathrm{~mm} \mathrm{Al} \mathrm{filter} \mathrm{of} 99 \%$ purity placed at the x-ray tube port. Air kerma was measured at the breast support platform as a function of tube mAs and corrected by the inverse square law to give the air kerma at the detector $(K)$; no correction was made for the transmission of detector covers. The detector response was then measured from uniform exposure (flood) images acquired as a function of air kerma at the detector. All detectors were fully irradiated (open collimation) for the flood acquisitions except for the Sectra MDM where a $12.8 \mathrm{~cm} \times 12.8 \mathrm{~cm}$ collimated field was used. For the systems with integrated flat-panel detectors the antiscatter grid was removed, while for CR systems the cassette was placed on the breast support platform. The target air kerma values were $12.5,25,50,100,200$ and $400 \mu \mathrm{Gy}$ at the detector, although this range could not be set for all systems. 'For Processing' DICOM images were then acquired; with the integrated flatpanel units, standard corrections for x-ray heel effect and detector offset, gain and defective 
pixels were applied. Pixel value (PV) and standard deviation were measured with an ROI of $5 \mathrm{~mm} \times 5 \mathrm{~mm}$ placed $60 \mathrm{~mm}$ from the chest wall edge and centred left-right on the detector. The standard deviation data were used for a basic separation of the system noise sources, described later. PV was then plotted against $K$ and one of three curves fitted to the data, depending on the dependence found:

$\mathrm{PV}=A+B \cdot K \quad$ or $\quad \mathrm{PV}=A+B \cdot \ln (K) \quad$ or $\quad \mathrm{PV}=A+B \cdot(K)^{0.5}$

For the slot scanning system, PV is directly related to the air kerma rate $\dot{K}$, which in turn is determined by the tube current and the scanning time:

$$
\mathrm{PV}=A+B \cdot \dot{K}
$$

All image data were converted to air kerma on a pixel-wise basis before analysis; this step linearized the image PV data, removed any offset that was present and gave images of unity gain.

\subsection{Detector non-uniformity}

Detector uniformity was calculated from the flood image acquired at an air kerma closest to $100 \mu \mathrm{Gy}$ and beam quality specified in table 2 . A rectangular ROI with sides chosen to be $1 \mathrm{~cm}$ smaller than each respective side for the image was positioned at the image centre and this region extracted and linearized. ROIs of dimension $1 \mathrm{~cm}^{2}$ were then taken from this extracted region and the mean, standard deviation and coefficient of variation (cov) calculated for the ROIs. The flood images were also inspected for specific artefacts at a narrow contrast setting.

\subsection{Modulation transfer function}

The pre-sampled MTF (Fujita et al 1992) was measured using a version of the edge method described by Samei et al (1998) implemented in the IDL (ITT Visual Solutions, Boulder, CO, USA) programming language (NHSBSP 2009b). For the $50 \mathrm{~mm} \times 50 \mathrm{~mm} \mathrm{MTF}$ tool, the edge was placed on the breast support platform or CR cassette such that the horizontal edge was $60 \mathrm{~mm}$ from the chest wall side and the vertical edge was centred left-right in the image. With the $60 \mathrm{~mm} \times 120 \mathrm{~mm}$ edge the MTF was calculated from two images acquired with the $120 \mathrm{~mm}$ edge at a distance of $60 \mathrm{~mm}$ from the chest wall edge, one image with the edge orientated horizontally and the second image edge orientated vertically, and centred left-right on the detector. For all MTF acquisitions, the edge was twisted such that there was an angle of approximately $3^{\circ}$ between edge and pixel matrix.

The edge images were acquired using the beam quality specified in table 2 , with a $2 \mathrm{~mm} \mathrm{Al} \mathrm{filter} \mathrm{at} \mathrm{the} \mathrm{x-ray} \mathrm{tube} \mathrm{port.} \mathrm{While} \mathrm{details} \mathrm{of} \mathrm{the} \mathrm{MTF} \mathrm{implementation} \mathrm{can} \mathrm{be} \mathrm{found}$ elsewhere (Marshall 2006a), some specific points are given here. A $45 \mathrm{~mm} \times 45 \mathrm{~mm}$ region of interest (ROI) was used to extract the edge ROI with the edge at the centre, which was then linearized to air kerma. The edge angle was estimated from a first-order least-squares fit to the maximum gradient calculated for the direction perpendicular to the edge transition. The edge angle was used to form a finely sampled edge spread function (ESF) from the PV data using the Crawford reprojection (Samei et al 1998) and a 5 pixel median filter was applied to the ESF. The ESF was differentiated to form the line spread function (LSF); the modulus of the fast Fourier transform (FFT) of the LSF was then normalized to the zero spatial frequency value to give the MTF. The two edge orientations were used to give front-back (across the detector) and left-right direction pre-sampling MTF curves. 


\subsection{Noise power spectrum}

The NPS was calculated from the detector response flood images, following the method prescribed by the International Electrotechnical Commission (IEC 2005). For the implementation here, a region of dimensions $1536 \times 1536$ pixels was extracted from the image centre and linearized to air kerma using the response curve. Region area was chosen to maximize the number of pixels in the evaluation, hence reducing uncertainty on the NPS, without adding additional error from increased non-uniformity. This was verified by examining the effect of region area on NPS and DQE for CR images, where large area non-uniformity is greater than that for flat-panel detectors. A second-order polynomial was then fitted to and subtracted from this region in order to reduce the influence of large area non-uniformity from sources such as the heel effect and detector non-uniformity on the NPS. Records of dimensions $256 \times 256$ pixels were taken from this region and the NPS formed by calculating the 2D FFT of each record using software written in IDL (NHSBSP 2009b). The records were half-overlapped by 128 pixels in the horizontal and vertical directions, giving 72 records from each image. Two methods were used to section a 1D NPS from the 2D ensemble; a radial average at full spatial frequency was used for systems with an isotropic NPS while for detectors with a non-isotropic NPS, the data were sectioned at full spatial frequency from 7 frequency bins (14 in total) on either side of the $0^{\circ}$ and $90^{\circ}$ NPS axes. Data from the $0^{\circ}$ and $90^{\circ}$ axes were included in the NPS estimate. The NNPS was then calculated by dividing by the square of the average PV for the linearized region i.e. by the square of the air kerma used for the flood image acquisition. Error on NPS was calculated following the method given by Dobbins et al (2006); with a radially sectioned NPS, the error is greater at low spatial frequencies as there are fewer points in the NPS.

\subsection{Detective quantum efficiency}

The DQE was calculated from the pre-sampled MTF and the NNPS (Dainty and Shaw 1974) using the equation

$$
\operatorname{DQE}(u)=\frac{\operatorname{MTF}^{2}(u)}{q_{0} \cdot K \cdot \operatorname{NNPS}(u)},
$$

where $q_{0}$ is found by integrating the x-ray photon spectrum and then normalizing for the air kerma to give photons per unit air kerma per $\mathrm{mm}^{2}$ for the $\mathrm{x}$-ray beam (calculated from the data of Boone et al (1997), and $K$ is the air kerma for the flood image acquisition. The true $q_{0}$ values depend on the properties of the actual x-ray photon spectrum for a given system and this in turn depends on the applied voltage waveform, tube target angle, tube potential and filtration composition, density, purity and linear thickness. Establishing this is not possible for field measurements such as these and hence the $q_{0}$ values used here are only approximate (in common with other studies). We note that the measured tube potential was within the remedial value $( \pm 1 \mathrm{kV})$ for all systems, and the estimated inherent filtration (filtration prior to the added $2 \mathrm{~mm} \mathrm{Al} \mathrm{filter)} \mathrm{was} \mathrm{within} \mathrm{manufacturer} \mathrm{tolerance} \mathrm{for} \mathrm{all} \mathrm{systems.} \mathrm{The} \mathrm{values}$ used for $q_{0}$ in this work are listed in table 2. The DQE was calculated as a function of air kerma for the detectors. For isotropic systems where the radial NNPS was used, the mean of the horizontal and vertical MTF curves was taken to form an average MTF. For non-isotropic NPS results, the $0^{\circ}$ and $90^{\circ}$ NNPS data were used in conjunction with the relevant MTF curve (horizontal and vertical). 


\subsection{Separation of system noise sources}

The standard deviation data measured in the detector response section were used to perform a basic separation of system noise sources similar to that described by Barnes (1982) for radiographic mottle in screen/film (S/F) detectors and applied to digital detectors by Borasi et al (2003) using standard deviation and Burgess (2004) using image variance. The standard deviation in the image was assumed to follow the relationship

$$
\sigma=\left(\sigma_{e}^{2}+\sigma_{q}^{2}+\sigma_{s}^{2}\right)^{0.5}
$$

where $\sigma_{e}, \sigma_{q}$ and $\sigma_{s}$ are the standard deviation terms representing electronic noise, quantum (x-ray photon) noise and structured noise, respectively. Note that this is a simplification and does not explicitly separate some aspects of detector noise, such as Poisson excess noise, secondary quantum noise and aliasing due to the sampling action of the pixel matrix. The contribution from each of these sources is included in the quantum noise term as these sources have the same dose (signal) dependence as primary quantum noise and hence cannot be separated using this formulation (Mackenzie and Honey 2007). This equation can be rewritten under the following assumptions. Electronic noise is an additive source and therefore should be independent of x-ray exposure and equal to some value, $\sigma_{e}=e$. Quantum noise scales with the square root of the air kerma (at a fixed beam quality and assuming Poisson noise), hence we can write $\sigma_{q}=q K^{0.5}$ for the quantum noise where $q$ is some coefficient. Structured 'noise' is in fact a deterministic pattern resulting from factors specific to a given detector, for example spatial gain variations of the x-ray convertor layer, large area nonuniformity from the x-ray beam (heel effect), scratches on the detector cover, dust and any non-uniformity added by the detector corrections. If known with sufficient precision then this pattern could be corrected; hence, this is not strictly an ergodic noise source although the pattern can potentially reduce the detectability of objects and hence can be thought of as a noise. The signal from these sources is amplified by the x-ray signal used; hence, we write $\sigma_{s}=s K$. Combining these terms gives the following for the standard deviation:

$$
\sigma=\left(e^{2}+q^{2} K+s^{2} K^{2}\right)^{0.5}
$$

For a given detector, standard deviation taken from the linearized flood image using a $5 \mathrm{~mm} \times 5 \mathrm{~mm}$ ROI was plotted as a function of air kerma at the detector; where possible the target air kerma range for the flood images was 12.5-400 $\mu \mathrm{Gy}$. A least-squares technique was used to determine the values for the fit coefficients $e, q$ and $s$.

One final test of detector noise was performed using linearized standard deviation plotted as a function of air kerma at the detector. An equation of the form

$$
\sigma=a \cdot K^{b}
$$

was fitted to the data; if image noise consists of pure Poisson (x-ray) noise across the exposure range examined then we expect coefficient $b$ to equal 0.5 . The presence of the additive and multiplicative detector noise sources discussed above will cause coefficient $b$ to deviate from 0.5 .

\section{Results and discussion}

\subsection{Detector response}

There was a $1.7 \%$ difference in measured output between the two dosemeters, averaged across the mAs values studied. No attempt was made to correct one set of output readings to match the other as we do not know which readings can be considered more accurate. Another option 
Table 3. MTF and DQE results for the systems studied; spatial frequency for the 50\% and $10 \%$ points of the MTF (averaged for the two directions unless specified); resolution anisotropy (ratio of spatial frequency of $50 \%$ MTF point (front-back to left-right); MTF at $5 \mathrm{~mm}^{-1}$ (averaged for the two directions unless specified); peak DQE and DQE at $5 \mathrm{~mm}^{-1}$.

\begin{tabular}{|c|c|c|c|c|c|c|}
\hline $\begin{array}{l}\text { Detector } \\
\text { name }\end{array}$ & $\begin{array}{l}\text { Spatial } \\
\text { frequency } \\
\text { for MTF } \\
50 \%\left(\mathrm{~mm}^{-1}\right)\end{array}$ & $\begin{array}{l}\text { Spatial } \\
\text { frequency } \\
\text { for MTF } 10 \% \\
\left(\mathrm{~mm}^{-1}\right)\end{array}$ & $\begin{array}{l}\text { Ratio spatial } \\
\text { frequency for } 50 \% \\
\text { MTF front-back } \\
\text { to left-right }\end{array}$ & $\begin{array}{l}\text { MTF at } \\
5 \mathrm{~mm}^{-1}\end{array}$ & $\begin{array}{l}\text { Peak } \\
\text { DQE }\end{array}$ & $\begin{array}{l}\mathrm{DQE} \text { at } \\
5 \mathrm{~mm}^{-1}\end{array}$ \\
\hline Agfa MM 3.0R & 1.99 & 5.82 & 1.12 & 0.13 & 0.36 & 0.07 \\
\hline Agfa HM 5.0 & 2.31 & 7.07 & 1.09 & 0.23 & 0.51 & 0.20 \\
\hline Fuji Profect—scan & 1.97 & 4.78 & 0.85 & 0.08 & 0.52 & 0.02 \\
\hline Fuji Profect-subscan & 2.31 & 5.58 & - & 0.14 & 0.54 & 0.07 \\
\hline Carestream EHR-M3 & 2.28 & 5.56 & 0.90 & 0.14 & 0.34 & 0.04 \\
\hline Fuji Amulet & 3.19 & 8.72 & 0.90 & 0.34 & 0.67 & 0.41 \\
\hline GE Senographe 2000D & 3.19 & 7.17 & 0.90 & 0.27 & 0.41 & 0.13 \\
\hline GE Senographe DS & 3.29 & 7.32 & 1.09 & 0.28 & 0.40 & 0.14 \\
\hline GE Essential & 2.38 & 6.07 & 1.03 & 0.16 & 0.59 & 0.18 \\
\hline Hologic Selenia & 4.53 & 10.1 & 1.02 & 0.44 & 0.48 & 0.21 \\
\hline Sectra MDM-orthoscan & 6.52 & 12.3 & 0.53 & 0.64 & 0.73 & 0.37 \\
\hline Sectra MDM-scan & 3.48 & 6.17 & - & 0.22 & 0.81 & 0.04 \\
\hline Siemens Inspiration & 4.86 & 9.58 & 1.11 & 0.48 & 0.44 & 0.15 \\
\hline
\end{tabular}

would be to increase/reduce (as appropriate) the dosemeter readings by $0.85 \%$; however, this is a very small correction and was not considered worthwhile. Tube potential and A/F settings used for the technical characterization are given in table 2; for the majority of systems these parameters were the same as those selected by the AEC device for $5 \mathrm{~cm}$ poly(methyl methacrylate) (PMMA) and used for the detectability calculations. The exceptions were the GE Senographe 2000D and DS systems where the technical characterization was performed at $28 \mathrm{kV}$ and Mo/Mo while the AEC selected $\mathrm{Rh} / \mathrm{Rh}$ and tube potentials of $28 \mathrm{kV}$ and $29 \mathrm{kV}$, respectively. Results for the detector response curve type and fitted A and B coefficients are listed in table 1. As expected, linear, logarithmic and power relationships were found for $\mathrm{PV}$ as a function of air kerma at the detector. The $r^{2}$ value (correlation coefficient) for the response curve fits was greater than or equal to 0.999 for all systems except the Fuji Amulet, where the $r^{2}$ was 0.975 indicating some deviation from the expected logarithmic $\left(r^{2}\right.$ increased when the higher $K$ values were excluded from the curve fit).

\subsection{Detector uniformity error}

Table 2 presents the detector uniformity error in terms of coefficient of variation for the 11 detectors. Note that no uniformity data are presented for the Sectra MDM as the flood images were physically collimated to $12.8 \mathrm{~cm} \times 12.8 \mathrm{~cm}$ for the acquisitions. As expected, the detectors formed two groups; higher uniformity error was seen for the CR cassette-based systems, with a range in cov from $9.1 \%$ to $18 \%$. Uniformity error for the flat-panel detectors was lower, varying from $1.2 \%$ to $2.7 \%$. We expect substantially lower uniformity error for the flat-panel detectors given the routine calibration of these detectors. Variations in x-ray signal (largely due to the heel effect) and detector sensitivity are measured as a function of area across the detector, x-ray signal (detector exposure) and beam quality. From these data, 
a calibration mask (Schmidgunst et al 2007) is formed that is used to correct signal and gain variations, reducing uniformity error (hence the term 'flat-field' correction is often applied to this procedure). These gain and non-uniformity corrections are not currently applied to cassette-based CR systems, leading to higher uniformity error. Close inspection under a narrow contrast setting revealed no artefacts for any of the detectors.

\subsection{Modulation transfer function}

Table 3 presents the spatial frequency for the $50 \%$ point of the pre-sampling MTF averaged in the front-back and left-right directions across the detector. Two exceptions are the Sectra MDM, where the MTF orthogonal and parallel to the scan direction is given and the Fuji Profect where MTF in the scan and subscan directions is given. For CR detectors read in a raster-type pattern using a laser, the scan direction refers to the direction in which the flying spot laser is guided rapidly across the CR phosphor plate while the sub-scan direction is the direction in which the plate is translated mechanically through the reader. As a measure of MTF isotropy, the ratio of the $50 \%$ point front-back to the spatial frequency for the $50 \%$ point for the left-right direction (scan direction for the Sectra MDM) results is given. This ratio shows less than $15 \%$ difference for nine detectors indicating reasonable MTF isotropy for most units; the exceptions are the Fuji Profect and the Sectra MDM systems. For the Sectra system, resolution is considerably higher in the direction orthogonal to the scan direction than in the scan direction. This is expected for the Sectra system and is due to motion blurring as the tube-detector assembly is translated across the breast platform (Åslund et al 2007). As a measure of limiting resolution, the spatial frequency at the $10 \%$ point of the MTF is also listed in table 3.

Given the number of systems, the pre-sampling MTF curves are presented as two separate groups: CR systems are plotted in figure 1(a), while the remaining digital radiography (DR) detectors (direct and indirect conversion flat-panel detectors and the Si-strip photon counter) are plotted in figure 1(b). These are averaged curves (front-back and left-right), again with the exception of the Fuji Profect and the Sectra MDM. Figure 1(a) shows remarkably consistent MTF performance between the different CR systems; this may suggest a similar thickness of powder/binder photostimulable phosphors used in this systems. Two MTF curves exhibit some differences: above $5 \mathrm{~mm}^{-1}$, the Agfa needle system has considerably higher MTF than the other CR systems which may reflect the reduced signal spread (blurring) for a needle phosphor compared to the powder phosphors. MTF for the Fuji Profect in the scan direction is somewhat lower than the other MTF curves above $3 \mathrm{~mm}^{-1}$. The reduced MTF in the scan direction has been explained in terms of the stimulated luminescence decay time and the scanning laser dwell time per pixel; resolution in the (fast) scan direction is affected when the dwell time is of the same order as the decay time (Rowlands 2002, Monnin et al 2006). These data can be compared against some results from the literature. While the energy does not have a large influence over the limited range of energies used for mammography, various MTF methodologies were used for the literature values, and this can have a substantial influence on the MTF curve (Carton et al 2005). MTF at $5 \mathrm{~mm}^{-1}$ in the work of Vandenbroucke and Leblans (2010) is approximately 0.20 and 0.18 , compared to 0.23 and 0.13 in table 3 . For the Fuji 5000MA (an early version of the dual-sided reading Fuji Profect CS), Fetterly and Schueler (2003) report approximate MTF values in the scan and subscan directions at $5 \mathrm{~mm}^{-1}$ of 0.12 , compared to 0.08 and 0.14 , respectively, for scan and subscan directions in table 3 .

Figure 1(b) plots average MTF curves for the DR-type systems, with the exception of Sectra MDM which is plotted as scan and orthogonal directions. For the sake of clarity just 

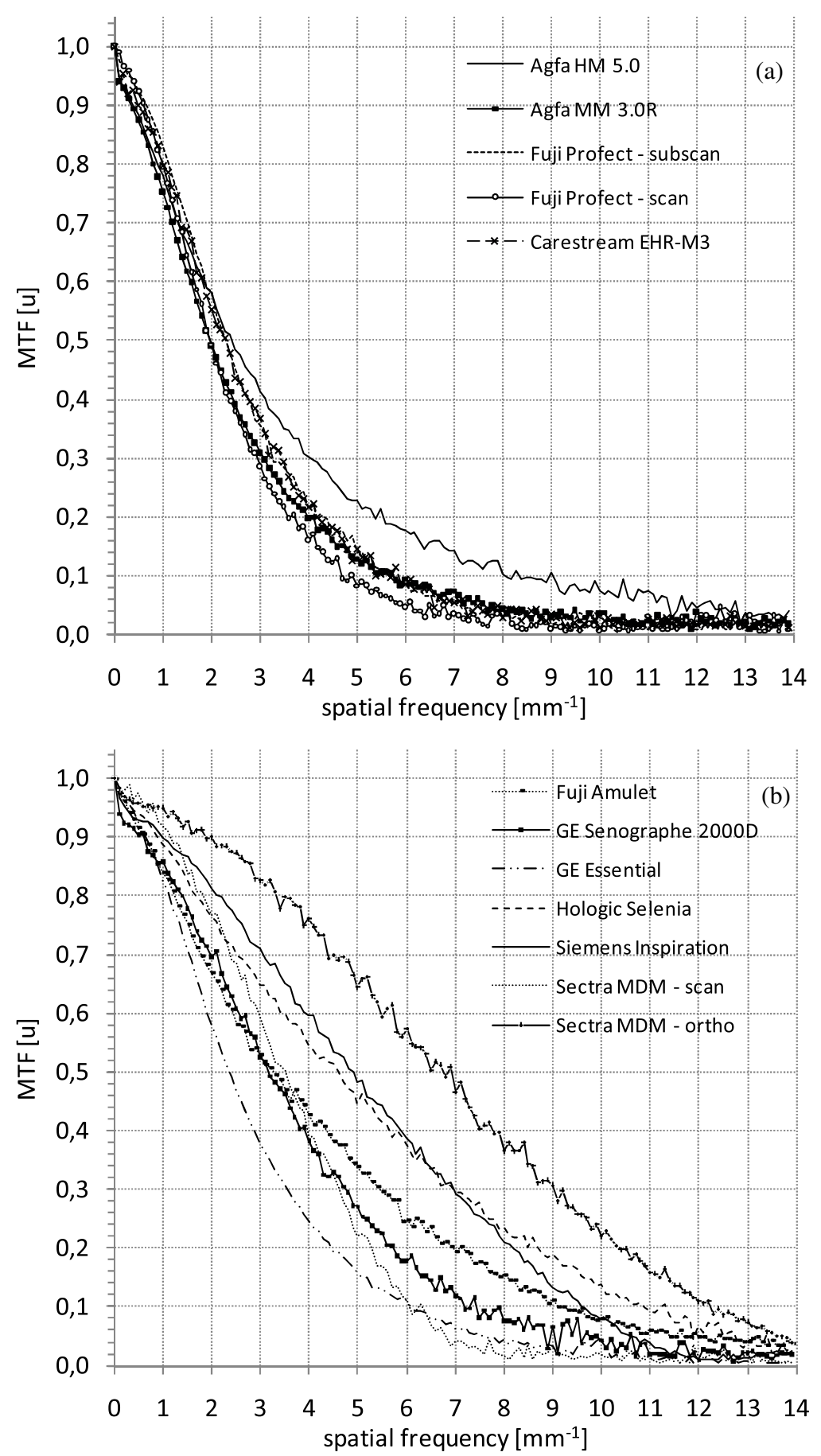

Figure 1. (a) Pre-sampling MTF for the CR detectors averaged in the left-right and front-back directions, with the exception of the Fuji Profect data, which are presented separately for the scan and subscan directions. (b) Pre-sampling MTF for the DR detectors averaged in the left-right and front-back directions, with the exception of the Sectra MDM data, which are presented separately for the scan and orthoscan directions. 
the GE Senographe 2000D results, rather than the GE Senographe DS data, are plotted in the following MTF, NNPS and DQE graphs. The same generation of detector is used in these systems and the results were almost equivalent; data for the GE Senographe DS are however given in the tables. A greater range of curves was found for these detectors. First, it can be seen that the pre-sampling MTF for the newer GE Essential detector is lower than the older generation detector used in the GE Senographe 2000D (and the GE Senographe DS). MTF at $5 \mathrm{~mm}^{-1}$ is 0.16 for the Essential compared to approximately 0.28 for the older generation detector. Pre-sampling MTF for the Fuji Amulet is close to the GE Senographe 2000D up to $4 \mathrm{~mm}^{-1}$ but then remains higher than the GE system at frequencies greater than $4 \mathrm{~mm}^{-1}$. Difference between the MTF for the $a$-Se-based Siemens Inspiration and Selenia Dimensions was small; however, MTF was higher for the Selenia above $8 \mathrm{~mm}^{-1}$, possibly reflecting the smaller pixel spacing of $70 \mu \mathrm{m}$ compared to $85 \mu \mathrm{m}$. We note that these MTF results are pre-sampling curves and include the intrinsic resolution of the x-ray converter layer sampled through the pixel aperture (which can be approximated by a sinc function that is directly related to the width of the sensitive region of the pixel (Zhao and Rowlands 1997, Rowlands and Yorkston 2000). For systems with little pre-sampling resolution loss, such as the two $a$ Se-based detectors here, MTF is expected to touch the spatial frequency axis at $1 / a$ where $a$ is the width of the pixel. MTF touches the axis at approximately $11.8 \mathrm{~mm}^{-1}$ (expect $11.8 \mathrm{~mm}^{-1}$ ) for the Siemens Inspiration while the MTF touches the spatial frequency axis at approximately $14.0 \mathrm{~mm}^{-1}$ (expect $14.3 \mathrm{~mm}^{-1}$ ). Both of these detectors have high pre-sampling MTFs at the respective Nyquist frequencies, with an MTF of 0.41 at $5.88 \mathrm{~mm}^{-1}$ for the Siemens unit and 0.29 at $7.14 \mathrm{~mm}^{-1}$ for the Selenia detector. Signals incident upon the detector containing substantial power at spatial frequencies above the respective Nyquist frequencies may be aliased, potentially leading to unpredictable behaviour when imaging small objects (Thomas et al 2005). Highest MTF was found for the Sectra MDM in the direction orthogonal to the scan motion (front-back) and hence considerable anisotropy exists for the Sectra unit; spatial frequency for $50 \%$ MTF in the scan direction is a factor of 2 lower than that for the direction orthogonal to the scan. It is worth noting that the CR systems have a pixel spacing of $50 \mu \mathrm{m}$ and yet generally have lower pre-sampling MTF curves than the flat-panel detectors, indicating that the $\mathrm{x}$-ray converter (photostimulable phosphor) is limiting resolution rather than the pixel dimension. It is possible that a larger pixel spacing could be used without reducing image quality.

Comparing data for MTF at $5 \mathrm{~mm}^{-1}$ against literature values, we found a value of 0.34 for the Fuji Amulet compared to the approximate figure of 0.45 for Rivetti et al (2009). For the GE Senographe 2000D, Vedantham et al (2000) give a figure of approximately 0.26 compared to the value of 0.27 found in this study. Results for the GE Senographe DS and the GE Essential were 0.28 and 0.16 ; these can be compared to figures of 0.38 and 0.15 in the study of Ghetti et al (2008). Results of 0.60 and 0.65 were found for an earlier version of the Hologic Selenia detector in studies by Monnin et al (2007) and Marshall (2009) compared to 0.44 given in table 3 for the newer detector (suitable for digital breast tomosynthesis). Åslund et al (2007) give results of 0.70 and 0.30 for the orthoscan and scan directions of the Sectra system; we found values of 0.64 and 0.22 for the orthoscan and scan directions, respectively. Finally, Oberhofer et al (2010) gave results of approximately 0.55 for a Siemens Inspiration unit, compared to the value of 0.48. Overall, the pre-sampling MTF results in this study are consistent with literature values taken from the previous 11 years, given differences in the various MTF conditioning/calculation methods and developments in the detectors themselves. 

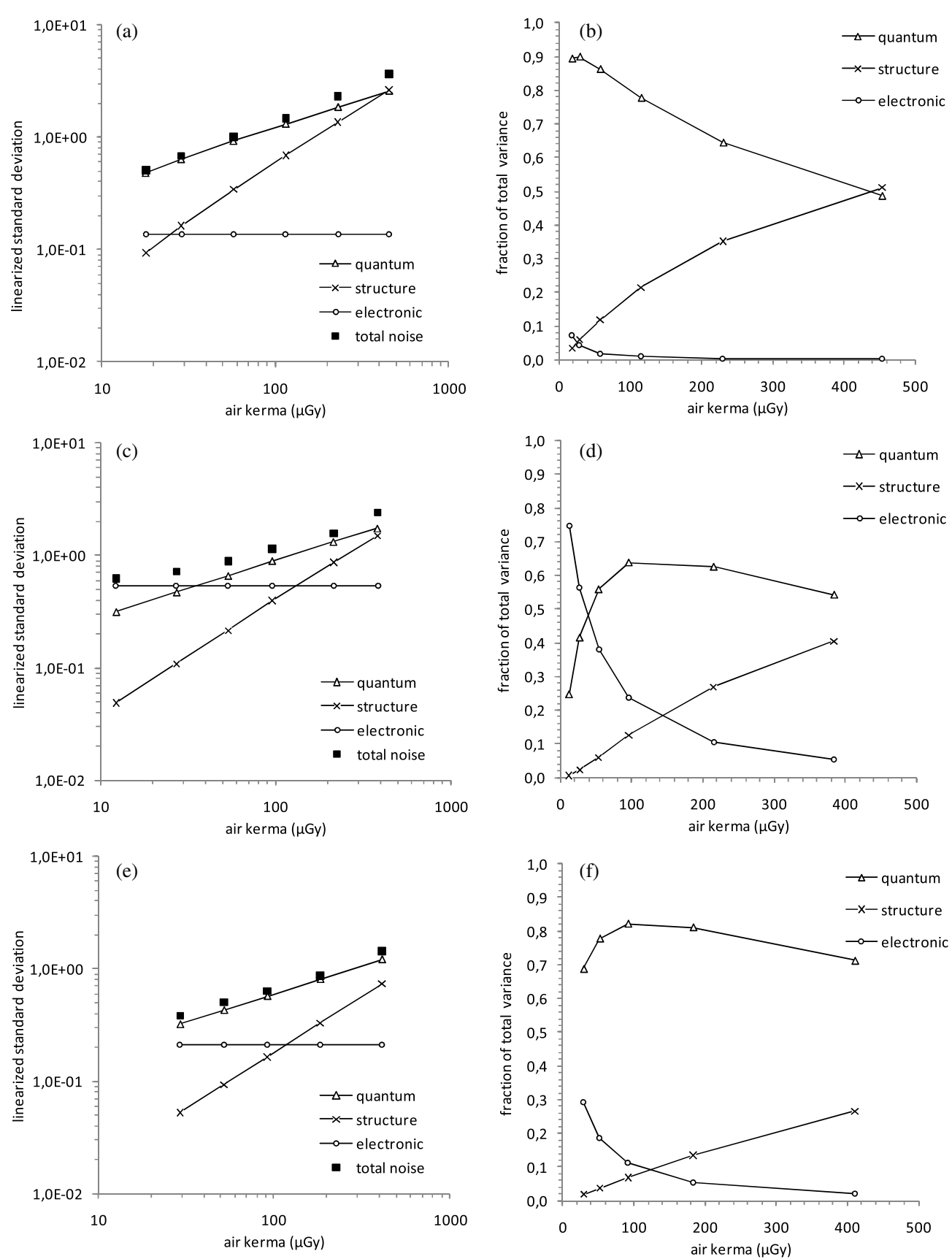

Figure 2. Linearized standard deviation representing total detector noise and separated electronic, $\mathrm{x}$-ray quantum noise and structure noise plotted as a function of detector air kerma for (a) the Agfa MM 3.0R CR detector, (c) the Fuji Amulet DR detector and (e) the GE Essential DR detector. Electronic, quantum and structured noise variance presented as a fraction of total noise variance for (b) the Agfa MM 3.0R CR detector, (d) the Fuji Amulet DR detector and (f) the GE Essential DR detector. 
Table 4. Approximate air kerma (lower and upper) where quantum noise is no longer the dominant noise component; approximate air kerma at the detector for the AEC mode; fit coefficient $b$ for standard deviation versus linearized PV.

\begin{tabular}{|c|c|c|c|c|}
\hline $\begin{array}{l}\text { Detector } \\
\text { name }\end{array}$ & $\begin{array}{l}\text { Lower } \\
\text { air kerma } \\
\text { bound } \\
(\mu \mathrm{Gy})\end{array}$ & $\begin{array}{l}\text { Upper } \\
\text { air kerma } \\
\text { bound } \\
(\mu \mathrm{Gy})\end{array}$ & $\begin{array}{l}\text { Air kerma } \\
\text { at detector } \\
\text { for AEC } \\
\text { mode }(\mu \mathrm{Gy})\end{array}$ & $\begin{array}{l}b \\
\text { coefficient }\end{array}$ \\
\hline Agfa MM 3.0R & $<18$ & $>450$ & 102 & 0.65 \\
\hline Agfa HM 5.0 & $<18$ & $>450$ & 104 & 0.60 \\
\hline Fuji Profect & $<124$ & $>384$ & 64 & 0.62 \\
\hline Carestream EHR-M3 & 32 & $>404$ & 65 & 0.61 \\
\hline Fuji Amulet & 40 & $>384$ & 88 & 0.39 \\
\hline GE Senographe 2000D & $<24$ & $>405$ & 66 & 0.49 \\
\hline GE Senographe DS & $<21$ & $>429$ & 98 & 0.49 \\
\hline GE Essential & $<29$ & $>411$ & 83 & 0.49 \\
\hline Hologic Selenia & $<13$ & $>390$ & 100 & 0.46 \\
\hline Sectra MDM & & & 64 & \\
\hline Siemens Inspiration & 22 & $>390$ & 98 & 0.33 \\
\hline
\end{tabular}

\subsection{Separation of noise sources}

Figure 2 plots the separated noise components as a function of air kerma for three selected systems: the Agfa MM 3.0R, the Fuji Amulet and the GE Essential. Both the linearized standard deviation and the fraction of each noise component compared to the total noise are plotted as a function of air kerma at the detector. The fractional approach was used for a digital fluorography system and allows the easy identification of the dominant noise source at a given air kerma, although previously the various noise sources were grouped together as a generic 'system' noise term (Marshall et al 2001). We note that while the noise sources can be separated using standard deviation or variance (Borasi et al 2003, Burgess 2004, European Commission 2006), if the results are to be expressed as a fraction of total noise then noise variance must be used. The noise components only remain additive and separable if expressed as a variance before calculating the relative value. From the noise model in equation $(2 b)$, additive noise (assumed to be electronic noise) dominates at low air kerma (x-ray signal), while structured noise becomes increasingly important at high air kerma. Two properties are expected for a well-designed detector: (a) quantum noise should be the highest noise component for the all detector air kerma values used clinically and (b) the magnitude of the additive and multiplicative noise sources should be low such that quantum noise forms a large fraction of the total noise, greater than or equal to $50 \%$ at least.

Table 4 presents the approximate quantum noise limited range for the detectors in this study. Electronic noise was sufficiently low for most detectors such that quantum noise remained the dominant noise at the lowest air kerma studied. Three exceptions were the Carestream EHR-M3 CR system, Fuji Amulet and the Siemens Inspiration, where electronic noise became the dominant component below approximately 32, 40 and $22 \mu \mathrm{Gy}$, respectively. Figures 2(c) and (d) present this graphically for the Fuji Amulet detector. Although not presented here, there was a reduction in DQE at low air kerma settings for these three detectors, which is consistent with the presence of electronic noise at low detector air kerma. The presence of electronic noise in a CR detector is unusual given that the photomultiplier tube used in these systems has a low dark current (Rowlands 2002). Operation of the CR detector at 
reduced gain could increase the relative importance of electronic noise at low exposure levels, an effect that has been demonstrated for flat-panel detectors (Schmidgunst et al 2007, Zhao 2007). This can be contrasted with the more typical result found for the Agfa MM 3.0R CR system; figures 2(a) and (b) show low electronic noise and structured noise that increases with exposure, approaching that of the quantum noise component at $400 \mu \mathrm{Gy}$. Figures 2(e) and (f) present similar graphs for the GE Essential detector. For this unit, quantum noise remains the dominant noise source for all the detector air kerma values examined; quantum noise variance as a fraction of total noise variance also remains between 0.7 and 0.8 for this air kerma range signifying good detector performance with respect to the additive and multiplicative noise sources.

Quantum noise remained the dominant noise for all detectors at $380 \mu \mathrm{Gy}$ or higher for all detectors. System AECs are generally programmed to aim for a target signal within the detector (in the form of PV) for a given breast thickness; this can be converted to some air kerma value at the detector using the detector response curve. For $50 \mathrm{~mm}$ PMMA, the air kerma at the detector under AEC control varied from 52 to $104 \mu \mathrm{Gy}$, with an average of $83.5 \mu \mathrm{Gy}$; this is shown in the accompanying paper (Monnin et al 2011). The figure of $380 \mu \mathrm{Gy}$ is therefore a factor of approximately 4.5 greater than the typical operating air kerma under the central region of the breast. Obviously air kerma will be lower in dense breast regions and higher towards the skin edge. Results in table 4 demonstrate that all the systems studied were quantum noise limited at the detector air kerma for the AEC operating point with the exception of the Fuji Profect. However, the data in table 4 show that the Fuji system was quantum noise limited at the lowest air kerma investigated (124 $\mu \mathrm{Gy})$ for this detector.

Table 4 presents the fitted $b$ coefficients for equation ( $3 c$ ) for the different systems. This table shows higher $b$ coefficients for two of the CR phosphor units $(b \sim 0.6)$, where structure noise often forms a higher proportion of system noise. Coefficients for the three GE systems and the Hologic Selenia unit are close to 0.5; quantum noise remained the dominant noise source for these systems over the exposure range studied. Lastly, for the Fuji Amulet and Siemens Inspiration units, $b$ is lower at 0.39 and 0.33 , respectively. Also, for the Carestream EHR-M3 CR system, $b$ was found to be 0.41 indicating high electronic noise at low detector air kerma, which is unusual for a CR system. This was confirmed by examining NNPS and $\mathrm{DQE}$ as a function of air kerma. From these results, we suggest that the presence of structure noise results in a $b$ coefficient greater than 0.5 for un-normalized noise, while electronic noise tends to reduce/flatten the exposure dependence of the total noise, leading to a $b$ coefficient considerably less than 0.5 . A further link is made in the second part of this study with regard to the gradient of the threshold contrast resolution plotted as a function of detector air kerma.

\subsection{Normalized noise power spectrum}

Target air kerma for the NNPS detector comparison was $100 \mu \mathrm{Gy}$; the largest difference was $24 \mu \mathrm{Gy}$ for the Fuji Profect system (124 $\mu$ Gy used)—see table 2 . This is radially averaged NNPS with the exception of the Fuji Profect and Sectra MDM detectors. For the radial NPS given here, error varied from $6.6 \%$ at $0.15 \mathrm{~mm}^{-1}$ to $1.4 \%$ at $5.0 \mathrm{~mm}^{-1}$. The error for axially sectioned NPS data was 3.0\%. NNPS was calculated as a function of air kerma for each detector. Given that the NNPS is inversely proportional to air kerma, figures 3(a) and (b) plots NNPS multiplied by the air kerma used for the flood image acquisition from which the NNPS was calculated. This removes the air kerma dependence between the different measurements; however, NNPS multiplied by air kerma will still vary between systems given the variation in DQE between detectors. Differences in $q_{0}$ as beam quality changes will introduce some additional variation. Figure 3(a) plots NNPS multiplied by air kerma for the CR cassette-based 
systems and shows a similar shape of NNPS across the CR systems. This is likely related to the MTF results, as similar pre-sampling MTF curves were seen between the CR units. MTF plays an important role in noise transfer for an imaging system, filtering both the primary X-ray noise and secondary quantum noise in the x-ray detector (Nishikawa and Yaffe 1990a, Mackenzie and Honey 2007). While of similar shape to other CR systems curves, the result for the Agfa MM 5.0 needle phosphor is lower, indicating lower noise for this CR phosphor. The result for the Fuji Profect is similar up to a spatial frequency of $4 \mathrm{~mm}^{-1}$; above this frequency NNPS increases then falls rapidly from $8 \mathrm{~mm}^{-1}$. This has been explained as the influence of a Butterworth filter on the photomultiplier data during read out in the scan direction, applied with the aim of reducing aliasing in the image (Kengyelics et al 1998, Rowlands 2002).

Figure 3(b) plots NNPS multiplied by air kerma for the DR-type detectors. Greater variation is seen in the shape of the NNPS for these systems; we also note greater variation between pre-sampling MTF curves for these detectors. The Sectra MDM, Selenia Dimensions and Siemens Inspiration have a flat NNPS, as expected (Rowlands and Yorkston 2000). For example, the ratio of NNPS at $5 \mathrm{~mm}^{-1}$ to NNPS at $1 \mathrm{~mm}^{-1}$ is 0.8 for the Sectra MDM and Siemens Inspiration units, an indication that there is limited blurring of the primary x-ray noise and secondary noise by the x-ray converters for these detectors (Nishikawa and Yaffe 1990b). Greater reduction in NNPS as a function of spatial frequency was seen for the GE detectors, with NNPS at $5 \mathrm{~mm}^{-1}$ compared to NNPS at $1 \mathrm{~mm}^{-1}$, dropping by factors of 0.20 and 0.12 for the GE Senographe 2000D/Senographe DS and GE Essential, respectively. This is probably a result of the X-ray converter blurring reducing quantum noise, leading to lower X-ray noise at higher spatial frequencies (Nishikawa and Yaffe 1990b). NNPS for the GE Essential detector is a factor of 2.0 and 3.4 lower than that for the GE Senographe 2000D/Senographe, at $1 \mathrm{~mm}^{-1}$ and $5 \mathrm{~mm}^{-1}$, respectively. This is despite being measured at a slightly higher $\mathrm{x}$-ray beam energy, where reduced absorption is expected to increase NNPS (Marshall 2009). The fact that the reduction in NNPS is greater at high spatial frequencies is due to the lower MTF and possibly a reduction in noise aliasing arising from greater pre-sampling blurring of the GE Essential detector.

\subsection{Detective quantum efficiency}

Table 3 lists peak DQE and DQE at $5 \mathrm{~mm}^{-1}$ for the 11 different systems; these data were calculated with an averaged MTF (left-right and front-back directions) and the radially averaged NNPS, unless indicated in the table. Air kerma and beam quality associated with these measurements is given in table 2. The full DQE curves are plotted in figures 4(a) and (b), interpolated to $0.25 \mathrm{~mm}^{-1}$ spacing using a linear interpolation. The two exceptions are the Fuji Profect and Sectra MDM, where the curves are kept separate for the scan and subscan or orthogonal scan directions. The CR data are plotted in figure 4(a), while the DR data are given in figure 4(b). For the CR group of detectors, highest peak DQEs are seen for the Fuji Profect and Agfa HM 5.0 needle phosphor systems. The DQE of the Fuji Profect is quite strongly peaked at low spatial frequencies, whereas the Agfa HM 5.0 maintains a high DQE at higher spatial frequencies (factor of approximately 4 greater at $5 \mathrm{~mm}^{-1}$ ). The Agfa HM5.0 needle phosphor had a higher DQE than the Agfa MM 3.0R and Carestream HER-M3 powder phosphors; peak DQE was approximately a factor of 1.5 greater while DQE at $5 \mathrm{~mm}^{-1}$ was higher by a factor of approximately 3.0. The lowest peak DQE of all the detectors was found for the Carestream EHR-M3 CR cassette at 0.34, while DQE at $5 \mathrm{~mm}^{-1}$ was 0.07 .

For the detectors in figure 4(b), largest peak DQE was found for the Sectra MDM at 0.81 and 0.73 for the scan and orthoscan directions, respectively. The influence of the non-isotropic MTF can be seen in the greatly differing DQE results at $5 \mathrm{~mm}^{-1}(0.37$ compared to 0.04$)$; it 

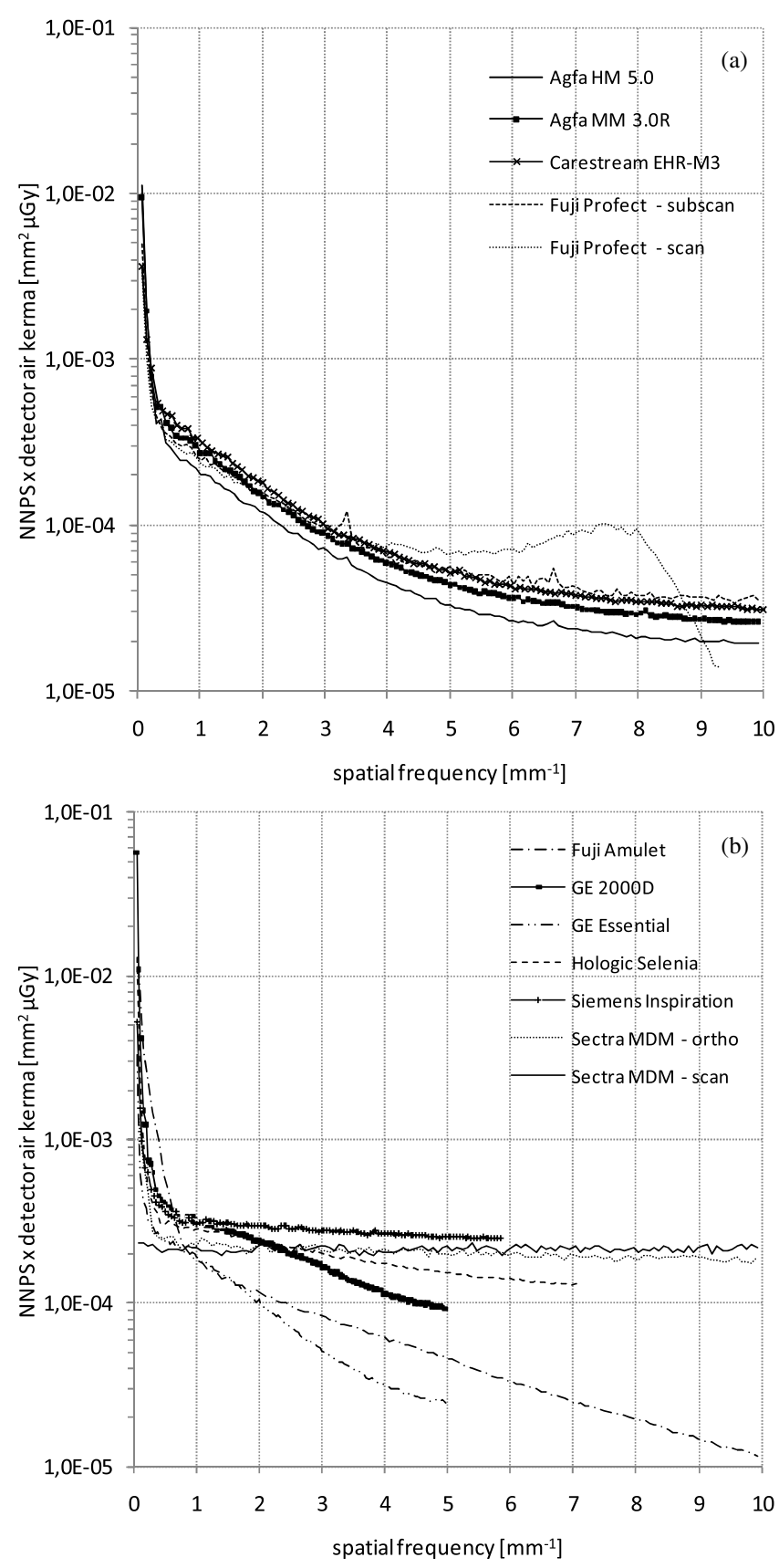

Figure 3. (a) Radially averaged NNPS for the CR detectors with the exception of the Fuji Profect data, which is presented separately for the scan and subscan directions. (b) Radially averaged NNPS for the DR detectors with the exception of the Sectra MDM data, which is presented separately for the scan and orthoscan directions.

is also possible that some structured noise has reduced DQE at low spatial frequency in the orthoscan direction (possibly from the structured strip detector assembly). The GE Essential has the next highest peak DQE in this group at 0.59 , which is a factor of 1.45 greater than the 

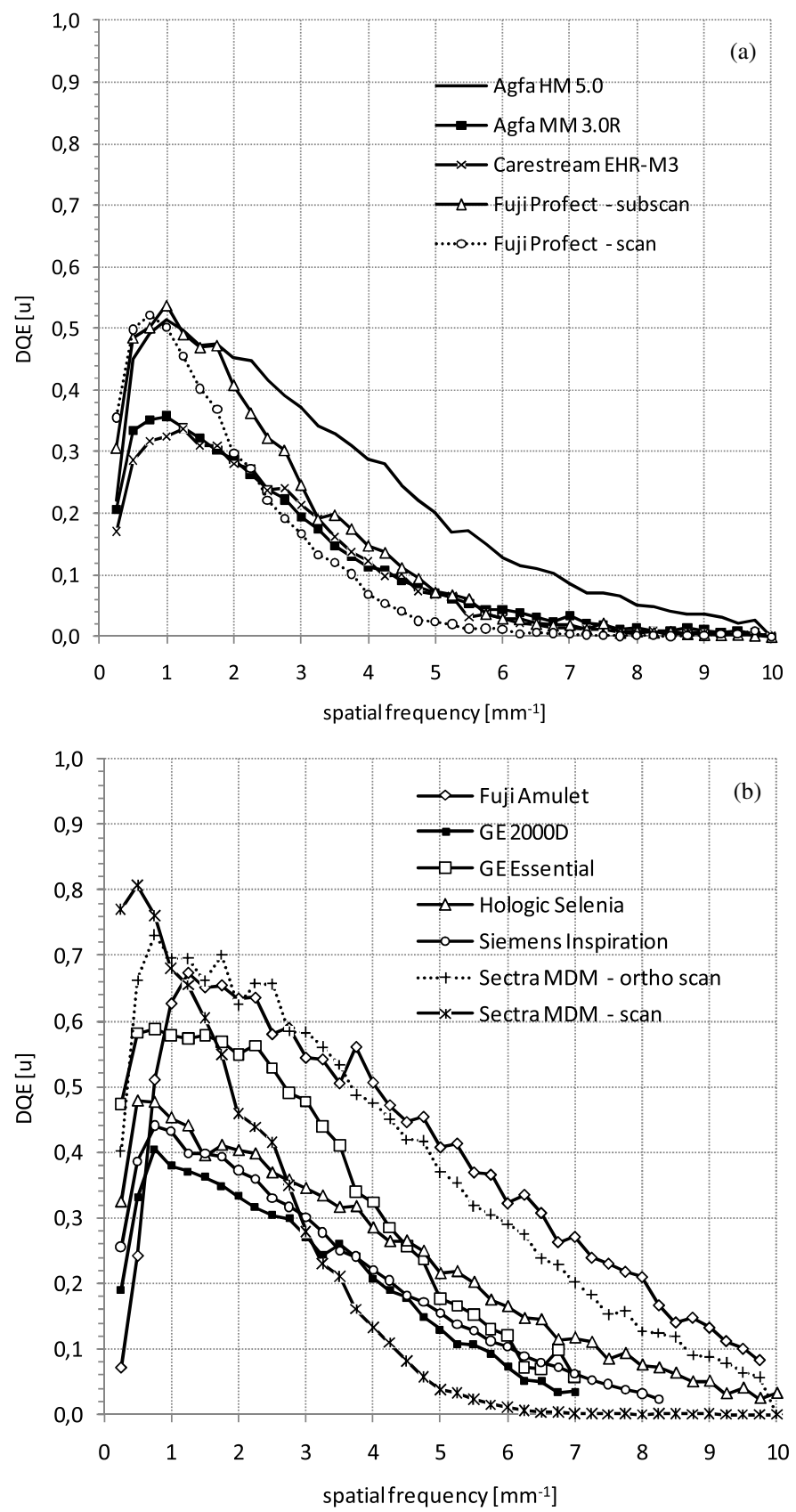

Figure 4. (a) DQE for the CR detectors and (b) DQE calculated for the DR detectors. Acquisition parameters are given in table 2. See the text for an explanation of the pre-sampling MTF and NNPS used in the calculation.

GE Senographe 2000D/Senographe DS detector result, with a peak DQE of approximately 0.41. The DQE results for the $a$-Se-based detectors are rather close, with a peak DQE of 0.48 and 0.44 for the Selenia Dimensions and the Siemens Inspiration, respectively. With the exception of the Agfa MM 5.0 needle phosphor CR system, the flat panel and photon 
counter-based units were able to maintain a high $\mathrm{DQE}$ at higher spatial frequencies compared to the $\mathrm{CR}$ systems. The ratio of DQE at $5 \mathrm{~mm}^{-1}$ to peak DQE for the powder CR detectors was approximately 0.18 against the average of 0.44 for the flat-panel detectors and a figure of 0.40 for the Agfa MM 5.0 needle phosphor. This indicates reduced signal-to-noise ratio transfer at high spatial frequencies is probably due to the poorer high-frequency MTF of the powder phosphors. While detector DQE influences object detectability (Aufrichtig 1999), other parameters such as the x-ray energy used for the acquisition (i.e. the contrast) and the quantity of scattered radiation will also play a major role. The second part of this study therefore examines the influence of low- and high-frequency DQE (typically peak DQE and DQE at $5 \mathrm{~mm}^{-1}$ ) on measured threshold detectability.

As with the MTF data, we can compare DQE (at $\sim 100 \mu \mathrm{Gy}$ ) against some literature values, although greater variation is expected for DQE owing to dosemeter calibration differences, the $\mathrm{MTF}^{2}$ squared dependence and the influence of NNPS conditioning, detector air kerma and beam quality on DQE. The data of Vandenbroucke and Leblans (2010), acquired at $28 \mathrm{kV} \mathrm{Mo} / \mathrm{Mo}$, show peak DQEs of 0.55 and 0.40 for the Agfa HM5.0 and MM3.0R, compared against 0.51 and 0.36 measured at $29 \mathrm{kV} \mathrm{Mo} / \mathrm{Rh}$ in this work. Fetterly and Schueler (2003) give a peak subscan DQE of approximately 0.58 measured at $81.7 \mu \mathrm{Gy}$ and $25 \mathrm{kV} \mathrm{Mo} / \mathrm{Mo}$ for the Fuji 5000MA, against a value of 0.54 measured at $27 \mathrm{kV}$ Mo/Rh for the Fuji Profect CS. Ghetti et al (2008) reported peak DQEs of approximately 0.58 and 0.48 for the GE Essential and GE Senographe DS, respectively, estimated from data acquired at $75 \mu \mathrm{Gy}$ and $28 \mathrm{kV}$ and a Mo/Mo A/F setting. For comparison, we found a peak DQE of 0.59 for the GE Essential and 0.40 for the GE Senographe DS. The study of Vedantham et al (2000) reports a peak DQE of 0.48 at $28 \mathrm{kV} \mathrm{Mo} /$ Mo with $40 \mathrm{~mm}$ PMMA for the GE Senographe 2000D, compared against the peak DQE of 0.41 in this work. An earlier study by Monnin et al (2007) gave peak DQEs of 0.40 and 0.40 for the Fuji Profect CR and Hologic Selenia DR systems, respectively ( $28 \mathrm{kV}$ and $\mathrm{Mo} / \mathrm{Mo}$ ); in this work we found figures of 0.54 and 0.48 , respectively. Åslund et al (2007) give peak DQEs of 0.72 and 0.73 in the scan and orthoscan directions, respectively, measured at $28 \mathrm{kV}$ using a W/Al A/F combination. Table 3 shows figures of 0.81 and 0.73 for the scan and orthoscan, respectively. Finally, Rivetti et al (2009) report a peak DQE of approximately 0.78 for the Fuji Amulet detector measured at $28 \mathrm{kV} \mathrm{W} / \mathrm{Rh}$ and $103 \mu$ Gy at the detector, which can be compared with the result of 0.67 in table 3 . Taken together, these data indicate that the systems assessed in the current study, using a common methodology, achieved similar performance in terms of DQE when compared against values reported in the literature.

\section{Conclusions}

This work examined a range of technical parameters for 11 mammography $\mathrm{x}$-ray detectors used in the detectability study, presented in the second part of the work. Results from the study can be summarized as follows. Detector uniformity error was notably lower for the flat-panel detectors (1.2\% to $2.7 \%$ ) compared to the CR detectors (9.1\% to $17 \%$ ), as might be expected given the large area gain corrections applied in these systems. MTF was isotropic to within $15 \%$ at the 50\% point of the MTF for most detectors, the exceptions being the Fuji Project and the Sectra MDM. Most detectors were quantum noise limited for the detector air kerma range of approximately 20-400 $\mu \mathrm{Gy}$, three exceptions being the Carestream EHR-M3 CR unit, the Fuji Amulet and the Siemens Inspiration DR detectors, where electronic noise dominated the image at low air kerma values. We could confirm that all systems were quantum noise limited at the detector air kerma for the AEC operating point; the exception was for the Fuji Profect was quantum noise limited at the lowest air kerma investigated for this detector of $124 \mu \mathrm{Gy}$. 
Peak DQE for detectors ranged from 0.34 for the Carestream EHR-M3 CR system to 0.81 for the Sectra MDM system in the scan direction. Reasonably close agreement was found between the peak DQE data of the systems in this study and data available in the literature; this is an indication that the systems evaluated were well set up and performing under what might be considered typical conditions.

\section{Acknowledgment}

We would like to thank Kim Lemmens for help in acquiring some of the data used in this paper.

\section{References}

Åslund M, Cederström B, Lundqvist M and Danielsson M 2007 Physical characterization of a scanning photon counting digital mammography system based on Si-strip detectors Med. Phys. 34 1918-25

Aufrichtig R 1999 Comparison of low contrast detectability between a digital amorphous silicon and a screen-film based imaging system for thoracic radiography Med. Phys. 26 1349-58

Barnes G T 1982 Radiographic mottle: a comprehensive theory Med. Phys. 9 656-67

Boone J M, Fewell T R and Jennings R J 1997 Molybdenum, rhodium, and tungsten anode spectral models using interpolating polynomials with application to mammography Med. Phys. 24 1863-74

Borasi G, Nitrosi A, Ferrari P and Tassoni D 2003 On site evaluation of three flat panel detectors for digital radiography Med. Phys. 30 1719-31

Burgess A 2004 On the noise variance of a digital mammography system Med. Phys. 31 1987-95

Carton A K, Vandenbroucke D, Struye L, Maidment A D, Kao Y H, Albert M, Bosmans H and Marchal G 2005 Validation of MTF measurement for digital mammography quality control Med. Phys. 32 1684-95

Dainty J C and Shaw R 1974 Image Science-Principles, Analysis and Evaluation of Photographic-Type Imaging Processes (London: Academic)

Dobbins J T III, Samei E, Ranger N T and Chen Y 2006 Intercomparison of methods for image quality characterization: II. Noise power spectrum Med. Phys. 33 1466-75

European Commission 2006 The European protocol for the quality control of the physical and technical aspects of mammography screening: part B. Digital mammography European Guidelines for Breast Cancer Screening 4th edn (Luxembourg: European Commission)

Fetterly K and Schueler B A 2003 Performance evaluation of a 'dual-side read' dedicated mammography computed radiography system Med. Phys. 30 1843-54

Fujita H, Tsai D Y, Itoh T, Doi K, Morishita J, Ueda K and Ohtsuka A 1992 A simple method for determining the modulation transfer function in digital radiography IEEE Trans. Med. Imaging 11 34-9

Ghetti C, Borrini A, Ortenzia O, Rossi R and Ordóñez P 2008 Physical characteristics of GE Senographe Essential and DS digital mammography detectors Med. Phys. 35 456-63

Hay G A, Clarke O F, Coleman N J and Cowen A R 1985 A set of X-ray test objects for quality control in television fluoroscopy Br. J. Radiol. 58 335-44

IEC (International Electrotechnical Commission) 2005 Medical electrical equipment — characteristics of digital x-ray image devices: part 1. Determination of the detective quantum efficiency IEC 62220-1-2 (Geneva: IEC)

Kengyelics S M, Launders J H and Cowen A R 1998 Physical imaging performance of a compact computed radiography acquisition device Med. Phys. 25 354-60

Mackenzie A and Honey I D 2007 Characterization of noise sources for two generations of computed radiography systems using powder and crystalline photostimulable phosphors Med. Phys. 34 3345-57

Marshall N W 2006a A comparison between objective and subjective image quality measurements for a full field digital mammography system Phys. Med. Biol. 51 2441-63

Marshall N W 2009 Detective quantum efficiency measured as a function of energy for two full-field digital mammography systems Phys. Med. Biol. 54 2845-61

Marshall N W, Kotre C J, Robson K J and Lecomber A R 2001 Receptor dose in digital fluorography: a comparison between theory and practice Phys. Med. Biol. 46 1283-96

Metz C E, Wagner R F, Doi K, Brown D G, Nishikawa R M and Myers K J 1995 Toward consensus on quantitative assessment of medical imaging systems Med. Phys. 22 1057-61

Monnin P, Gutierrez D, Bulling S, Guntern D and Verdun F R 2007 A comparison of the performance of digital mammography systems Med. Phys. 34 906-14 
Monnin P, Holzer Z, Wolf R, Neitzel U, Vock P, Gudinchet F and Verdun F R 2006 An image quality comparison of standard and dual-side read CR systems for pediatric radiology Med. Phys. 33 411-20

Monnin P, Marshall N W, Bosmans H, Bochud F O and Verdun F R 2011 Image quality assessment in digital mammography: part II. NPWE as a validated alternative for contrast detail analysis Phys. Med. Biol. 56 4221-38

NHSBSP (National Health Service Breast Screening Programme) 2009a Commissioning and routine testing of full field digital mammography systems NHSBSP Equipment Report 0604 (Sheffield: NHSBSP Publications)

NHSBSP (National Health Service Breast Screening Programme) 2009b Calculation of quantitative image quality parameters NHSBSP Equipment Report 0902 (Sheffield: NHSBSP Publications)

Nishikawa R M and Yaffe M J 1990a Effect of various noise sources on the detective quantum efficiency of phosphor screens Med. Phys. 17 894-904

Nishikawa R M and Yaffe M J 1990b Model of the spatial-frequency-dependent detective quantum efficiency of phosphor screens Med. Phys. 17 887-93

Oberhofer N, Fracchetti A, Nassivera E, Valentini A and Moroder E 2010 Comparison of two novel FFDM systems with different a-Se detector technology: physical characterization and phantom contrast detail evaluation in clinical conditions Digital Mammography 10th Int. Workshop, IWDM 2010 (Girona, Spain) ed J Martí, A Oliver, J Freixenet and R Martí (Berlin: Springer) pp 459-66

Rivetti S, Lanconelli N, Bertolini M, Borasi G, Golinelli P, Acchiapati D and Gallo E 2009 Physical and psychophysical characterization of a novel clinical system for digital mammography Med. Phys. 36 5139-48

Rowlands J A 2002 The physics of computed radiography Phys. Med. Biol. 47 R123-66

Rowlands J A and Yorkston J 2000 Flat panel detectors for digital radiology Physics and Psychophysics vol 1 ed J Beutel, H L Kundel and R L Van Metter (Bellingham, WA: SPIE Optical Engineering Press) pp 223-328

Samei E and Flynn M J 2003 An experimental comparison of detector performance for direct and indirect digital radiography systems Med. Phys. 30 608-22

Samei E, Flynn M J and Reimann D A 1998 A method for measuring the presampled MTF of digital radiographic systems using an edge test device Med. Phys. 25 102-13

Samei E, Ranger N T, Dobbins J T III and Chen Y 2006 Intercomparison of methods for image quality characterization: I. Modulation transfer function Med. Phys. 33 1454-65

Schmidgunst C, Ritter D and Lang E 2007 Calibration model of a dual gain flat panel detector for 2D and 3D x-ray imaging Med. Phys. 34 3649-64

Thomas J A, Chakrabarti K, Kaczmarek R and Romanyukha A 2005 Contrast-detail phantom scoring methodology Med. Phys. 32 807-14

Vandenbroucke D A N and Leblans P J R 2010 CR mammography: image quality measurement and model calculation for needle versus powder imaging plate Digital Mammography 10th Int. Workshop, IWDM 2010, (Girona, Spain) ed J Martí, A Oliver, J Freixenet and R Martí (Berlin: Springer) pp 216-26

Vedantham S et al 2000 Full breast digital mammography with an amorphous silicon-based flat panel detector: physical characteristics of a clinical prototype Med. Phys. 27 558-67

Zhao B 2007 Breast tomosynthesis with amorphous selenium digital flat panel detector PhD Thesis Stony Brook University

Zhao W and Rowlands J A 1997 Digital radiology using active matrix readout of amorphous selenium: theoretical analysis of detective quantum efficiency Med. Phys. 24 1819-33 